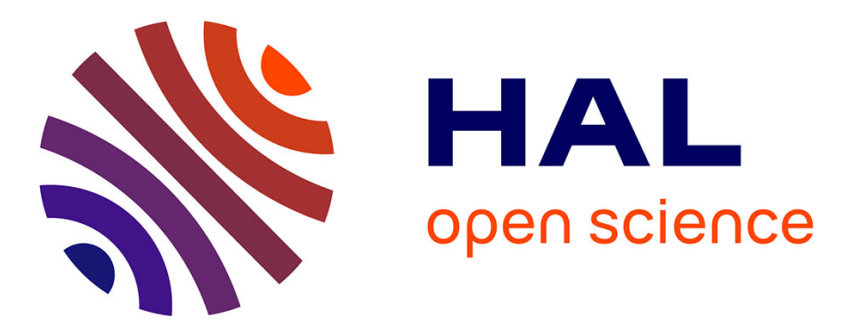

\title{
Structural exploration and Förster theory modeling for the interpretation of gas-phase FRET measurements: Chromophore-grafted Amyloid- $\beta$ peptides
}

Alexander Kulesza, Steven Daly, Luke Macaleese, Rodolphe Antoine, Philippe Dugourd

\section{To cite this version:}

Alexander Kulesza, Steven Daly, Luke Macaleese, Rodolphe Antoine, Philippe Dugourd. Structural exploration and Förster theory modeling for the interpretation of gas-phase FRET measurements: Chromophore-grafted Amyloid- $\beta$ peptides. Journal of Chemical Physics, 2015, 143 (2), pp.025101. 10.1063/1.4926390 . hal-01344520

\author{
HAL Id: hal-01344520 \\ https://hal.science/hal-01344520
}

Submitted on 12 Jul 2016

HAL is a multi-disciplinary open access archive for the deposit and dissemination of scientific research documents, whether they are published or not. The documents may come from teaching and research institutions in France or abroad, or from public or private research centers.
L'archive ouverte pluridisciplinaire HAL, est destinée au dépôt et à la diffusion de documents scientifiques de niveau recherche, publiés ou non, émanant des établissements d'enseignement et de recherche français ou étrangers, des laboratoires publics ou privés. 


\title{
Structural exploration and Förster theory modeling for the interpretation of gas-phase FRET measurements: Chromophore-grafted Amyloid- $\beta$ peptides
}

\author{
Alexander Kulesza, ${ }^{1}$ Steven Daly, ${ }^{1}$ Luke MacAleese, ${ }^{1}$ Rodolphe Antoine, ${ }^{1}$ and Philippe Dugourd ${ }^{1}$ a) \\ Institut Lumière Matière, UMR5306 Université Claude Bernard Lyon 1-CNRS, Université de Lyon, \\ 69622 Villeurbanne cedex, France
}

(Dated: 20 June 2015)

The distance-dependence of excitation energy transfer, e.g. being described by Förster theory (FRET), allows the use of optical techniques for the direct observation of structural properties. Recently, this technique has been successfully applied in the gas phase. The detailed interpretation of the experimental FRET results, however, relies on the comparison with structural modeling. We therefore present a complete first-principles modeling approach that explores the gas-phase structure of chromophore-grafted peptides and achieves accurate predictions of FRET efficiencies. We apply the approach to Amyloid- $\beta$ 12-28 fragments, known to be involved in amyloid plaque formation connected to Alzheimer's disease. We sample stuctures of the peptides that are grafted with 5-carboxyrhodamine 575 (Rh575) and QSY-7 chromophores by means of replicaexchange molecular dynamics simulations upon an Amber-type forcefield parametrization as a function of the charge state. The generated ensembles provide chromophore-distance and -orientation distributions which are used with the spectral parameters of the Rh575/QSY-7 chromophores to model FRET-efficiencies for the systems. The theoretical values agree with the experimental average "action"-FRET efficiencies and motivate to use the herein reported parametrization, sampling and FRET-modeling technique in future studies on the structural properties and aggregation-behavior of related systems.

PACS numbers: 33.50.-j, 87.10.Tf, 87.14.ef, 87.15.A-

\section{INTRODUCTION}

The investigation of protein structure and aggregation behavior is integral to understand protein function and dysfunction and in particular protein misfoldingrelated diseases ${ }^{1}$. For example, the first stages of how the amyloid plaques of Alzheimer patients are formed and what causes this aggregation to happen is not yet fully understood. To tackle this quest, global structuredetermination methodologies have to evolve that allow monitoring structural parameters of the precursor molecules and to observe misfolding at the molecular level. The gas phase provides a well-suited environment to implement such global strategy as the conditions for the experiments can be precisely defined and the overall structure of complex molecular edifices can be preserved using soft ionization techniques ${ }^{2,3}$.

Förster resonance energy transfer (FRET) is a phenomenon that allows the probing of structural parameters by means of optical techniques. In the picture of Förster Theory developed in the late forties ${ }^{4}$, the efficiency for a resonant energy transfer between the chromophores depends on their mutual distance as well as orientation of their transition dipole moments. FRET is usually measured by recording a decrease in the fluorescence of the donor chromophore, with the concurrent emergence of the acceptor's fluorescence or by changes in fluorescence decay times. The distance dependence is described by Förster's approximation, (equation 1)

${ }^{a)}$ Electronic mail: philippe.dugourd@univ-lyon1.fr where the so-called critical Förster distance $\mathrm{R}_{0}$ defines the distance at which $50 \%$ of the donor excitation is transferred to the acceptor moiety. It is routinely applied as a model for donor-acceptor distances on a length scale from 1 to $10 \mathrm{~nm}$ and when dipole-dipole (or convergent multipole-multipole) approximation for the electronic coupling can be employed appropriately for the donor-acceptor interaction ${ }^{5}$.

$$
E_{F R E T}=\frac{1}{1+\left(r / R_{0}\right)^{6}}
$$

The energy transfer is dominated by the $\frac{1}{r^{6}}$ distancedependence that imprinted the term "spectroscopic ruler" 6 on the technique. Thus, the interpretation of FRET results relies on the possibility to graft specific chromophores at well-defined sites on a molecule. Taking into account the flexibility of the chromophores and their linkers, the measured intensities provide information on the mutual distance of these specific sites ${ }^{7-10}$ e.g. to monitor structural changes in biomolecules ${ }^{6,11}$.

The use of multiple dye pairs allows for triangulation of biomolecules, which provides three-dimensional structural information ${ }^{9,12-16}$. Consequently, combining this phenomenon with the concept of single molecule detection $^{17-19}$ and time-resolved experiments ${ }^{20}$ allows for obtaining a unique glimpse into molecular structure as well as inter- and intra-molecular motions. While there exist numerous applications of FRET in the condensed phase $^{21-24}$, gas-phase FRET measurements ${ }^{25-34}$ suffer from difficulties in recording the fluorescence of a small number of ions in a mass spectrometer. The development of techniques capable of probing FRET in the gas 
phase with higher sensitivity is thus of high interest. This FRET detection problem has been recently overcome by combining photofragmentation action-spectroscopy with FRET ("action"-FRET) 35 , opening up the possibility to integrate gas-phase FRET into a global approach for structure determination of proteins and protein complexes ${ }^{36-38}$.

Extracting exact distances from an experimental calibration of FRET efficiencies alone is difficult because additional structural parameters like the orientation of the chromophore's transition dipole moments affect the excitation energy transfer efficiency significantly. In fact, experimental single-molecule FRET (smFRET) studies on DNA strands allowed to specify those orientations and showed a clear orientation-modulation ${ }^{39,40}$ indicating that a negligence of the explicit orientational distribution in "fixed conformations" limits the predictive power. Even in systems where the dye orientation is not strongly restricted, a significant influence on the overall FRET-efficiency was suggested ${ }^{41}$.

Therefore, modeling efforts that access the structure of the chromophore-grafting sites and their distribution or temporal evolution are most attractive. These serve as the basis for the calculation of excitation energy transfer efficiencies and thus assign a detailed molecular picture to the experiments. The accuracy of FRET-efficiency prediction is limited by two major factors. Firstly, the Förster formula is straightforward in its use, but it relies on the validity of its underlying assumption of dipoledipole interaction. Excitation energy transfer rates based calculated with approaches beyond Förster Theory ${ }^{5}$ can overcome this issue. For a recent review on the calculation of electronic coupling in excitation energy transfer see ref. ${ }^{42}$. In particular, nonadiabatic dynamics ${ }^{43,44}$ or semiclassical treatments ${ }^{45,46}$ of the excited state dynamics could deliver accurately the excitation energy transfer efficiencies (though at higher computational costs). Secondly, the prediction's accuracy depends critically on the reliability of the structural exploration - being the underlying foundation for the subsequent numerical modeling. Notably, Hoefmüller et al. recently combined single molecule FRET experiments with the mutual dye orientation statistics obtained from Molecular Dynamics (MD) simulations. From the simulated timedependent mutual orientations and distances, FRET efficiencies were calculated and the full statistics of individual photon absorption, energy transfer, and photon emission events were obtained from subsequent Monte Carlo (MC) simulations of the FRET kinetics ${ }^{47}$. The technique is dedicated to time-coherent single-molecule studies in solution and its algorithms and implementation are part of a computational toolkit for the simulation of smFRET on the basis of molecular dynamics (MD) trajectory ensembles ${ }^{48}$. Dedicated to gas-phase FRET, Talbot et al. have combined gas-phase fluorescence measurements and MD-based numerical modeling of the critical Förster distance ${ }^{27}$. The qualitative insight from the calculations allowed them to interpret molecu- lar structures on basis of their modeled conformations. However, conventional MD may not overcome barriers between the guessed initial configuration and the global minimum and may therefore fail to achieve sampling of a representative structural basis. The resulting errors in the modeling of FRET-efficiencies can be qualitative in nature also using accurate methods for excitation energy transfer calculations and can be overcome only with techniques that enhance the exploration of conformational space.

Such an enhanced structural sampling of gas-phase chromophore-grafted peptides together with numerical modeling of FRET-efficiencies based on distances and orientations at the same time has not yet been reported. In this work, we devise a strategy that succeeds in the accurate numerical first-principles modeling of FRET efficiencies based on a thorough exploration and enhanced sampling of gas-phase structures. In brief, we exploit Replica-Exchange Molecular Dynamics $^{49}$ (REMD) for the sampling procedure that offer a convenient framework to gas-phase conformations and statistical analyses ${ }^{50}$. Taking into account the distance distributionss and chromophore-orientation distributions at a given temperature, we calculate averaged FRET-efficiencies according to Förster-Theory and finally compare the results with experimentally measured "action"-FRET efficiencies published elsewhere ${ }^{51}$. The paper is structured as follows: we first describe the model peptide and chromophores used throughout this paper, then we explain our modeling setup detailing the procedure to obtain structural ensembles by means of REMD. Finally, we report the numerical modeling of FRET efficiencies and compare them to their experimental counterparts.

\section{RESULTS AND DISCUSSION}

\section{A. System and experimental gas-phase FRET}

In order to establish our strategy for converging "action-FRET" experiments and REMD ensemble based FRET modeling, we chose the Amyloid- $\beta$ 12-28 fragment with the sequence $\mathrm{V}^{12}$ HHQKLVFFAEDVGSNK ${ }^{28}$ for which we recently published experimental data ${ }^{52}$. It has been shown that the naturally occurring mutations in the hydrophobic cluster (residues 17-22) are an important factor of Amyloid- $\beta$ aggregation and which are probably indicating an enhanced risk of developing Alzheimer's disease ${ }^{53-55}$. We used the FRET-donoracceptor pair that we have already proven suitable for this purpose $^{35}$. The acceptor chromophore is QSY7C5 maleimide, a dark quencher absorbing and fragmenting efficiently at $545 \mathrm{~nm}$ in the gas phase ${ }^{35,56}$. The donor chromophore is 5-carboxyrhodamine 575 (Rh575) C5 maleimide, absorbing at $505 \mathrm{~nm}$ in the gas phase. Rh575 was also used as the donor chromophore by Talbot et $a l^{27}$, and both the fluorescence excitation 

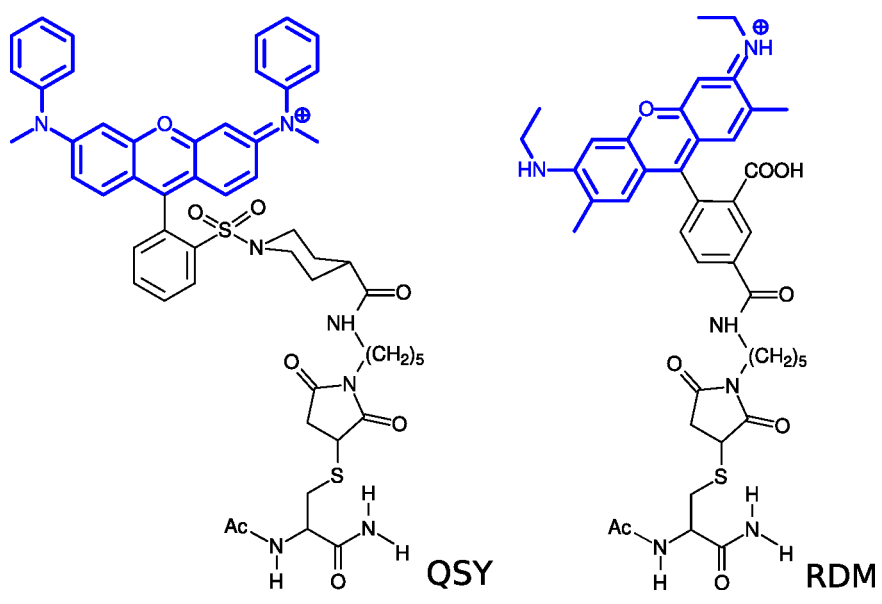

FIG. 1. Chemical formulas of the QSY7-C 5 maleimide and Rhodamine $575-\mathrm{C}_{5}$ maleimide chromophores grafted to a Accysteine- $\mathrm{NH}_{2}$ peptide used for the parametrization of the nonstandard aminoacid residues (denoted as RDM and QSY residues in the following).

and emission spectra have been previously studied in detail $^{57}$. Both chromophores exhibit a C5 linker chain and a terminal maleimide group that allows thiol-specific grafting under mild conditions. The grafting does not affect significantly the absorption and emission properties of the chromophores. As the target peptide must contain 2 thiol groups for the grafting, our analyte molecule exhibits capped $\mathrm{N}$ - and C-terminal cysteine residues (Ace-CV ${ }^{12} \mathrm{HHQKLVFFAEDVGSNK}{ }^{28} \mathrm{C}$ $\mathrm{NH}_{2}$ ). The chemical formulas of the chromophores grafted onto the cysteine residues are depicted in figure 1. The QSY7-C5 maleimide and Rh575-C5-maleimide grafted cysteine residues will be denoted throughout the paper as QSY and RDM, respectively. Complementing the herein established theoretical technique, an experimental-theoretical study of the gas-phase structure of chromophore-grafted Amyloid- $\beta$ peptides is published elsewhere $^{51}$.

\section{B. Computational setup and structural sampling}

Replica Exchange Molecular Dynamics (REMD) simulations $^{49}$ were conducted to obtain structural ensembles for the chromophore grafted Amyloid- $\beta$ peptides. The peptide was described on basis of the the Amber $99^{58}$ force field, that, different to more recent versions, was parametrized against gas-phase ab-initio calculations. It was completed with the Generalized Amber Force Field $\left(\mathrm{GAFF}^{59}\right)$ parametrizing the chromophore grafted cysteine residues QSY and RDM. GAFF is designed to "interoperate" with the Amber force field and is suitable for a broad variety of organic molecules. The dye residues were constructed by first finding a low-energy gas-phase conformation of Ace-QSY-NH 2 and Ace-RDM$\mathrm{NH}_{2}$ molecules. For this purpose we ran Molecular Dy- namics (MD) with simulated annealing based on forces obtained with the semiempirical AM1 Hamiltonian ${ }^{60}$ using a local code. To obtain the GAFF parameter sets we exploited AM1-bcc ${ }^{61}$ calculations that reproduce $\mathrm{HF} / 6$ $31 \mathrm{G}^{*}$ RESP charges. We used the software acpype ${ }^{62}$ in conjunction with Antechamber of the AmberTools version 14 suite of programs ${ }^{63}$ for this process. From the parameter sets, we created nonstandard internal residues by removing either the Ace or the $\mathrm{NH}_{2}$ cap and applying manually a small offset to the partial charges of the connecting atoms in order to re-establish the correct total charge of the residues. The residue libraries in Amber format can be obtained from the supplemental material. The interface between the GAFF and Amber99 residues was achieved by assigning Amber99 amino acid bond-, angle- and dihedral-parameters of the interfacing peptide bond.

All forcefield MD simulations and optimizations were carried out with the GROMACS suite of software in its version $4.6 .5^{64-66}$. Topologies of the chromophoregrafted Amyloid beta peptides in $3+, 4+, 5+$ and $6+$ charge states were constructed using the Leap program of the Ambertools distribution and converted to GROMACS topologies with acpype. The Ace$\mathrm{CV}^{12} \mathrm{HHQKLVFFAEDVGSNK}{ }^{28} \mathrm{C}-\mathrm{NH}_{2}$ peptides were created in a full extended conformation and the cysteine residues mutated to QSY and RDM in both possible sequences. Additional to the fixed charges from the chromophores (amounting to +2 ), one, two or three protons were distributed over the LYS (in priority) and HIS residues (LYS 16, LYS 28, HIS 13, HIS 14) and by using the respective parameter set of protonated or neutral residues. For the $3+$ and $5+$ system, two different topologies resulting from the different possible locations of the proton were obtained. ASP and GLU residues were treated in their neutral form (ASH and GSH Amber residue types) since for relatively small peptides zwitterionic structures are generally less stable in the gas phase. For the MD simulations the systems were treated nonperiodically without solvent molecules or implicit solvation and with no cutoff for nonbonded interactions. For optimizations, the L-BFGS algorithm was employed until a potential energy convergence of $1 \mathrm{~kJ} / \mathrm{mol}$ was achieved.

MD trajectories were propagated using the velocityverlet integrator with a timestep of $0.001 \mathrm{ps}$. Thermalization was achieved via coupling with an external heat bath in frame of the velocity-rescaling algorithm ${ }^{67}$ that incorporates a stochastic term and thus provides the correct canonical ensemble. Replica exchange molecular dynamics simulations as implemented in GROMACS were conducted by propagating 20 trajectories in parallel. The temperatures of the replicas span a range of $220 \mathrm{~K}$ to $850 \mathrm{~K}(220,236,254,272,292,314,337,362,389,417$, $448,481,517,555,596,640,687,737,792,850)$. After a $0.1 \mathrm{~ns}$ equilibration of the replica at their temperatures, sampling of the structures with REMD was begun. A REMD simulation was conducted with a length of $9.9 \mathrm{~ns}\left(9.9 \cdot 10^{6}\right.$ steps $)$ totaling to $10 \mathrm{~ns}$ of MD trajec- 
tory in all temperatures. Exchanges between adjacent replica were attempted every 200 integration steps. The chosen temperatures gave rise to exchange probabilities of 0.01 to 0.1 that ensured the mobility of the replicas through the whole temperature range. After a completed REMD sampling, the $220 \mathrm{~K}$ trajectory was quenched by extracting and optimizing every $1000^{\text {th }}$ step to the next local minimum. A new calculation cycle was started with the found lowest-energy (LE) structure as starting point. The convergence of the procedure was monitored by the energy of the optimized LE structure and the potential energy distribution function (PEDF) of the $220 \mathrm{~K}$ and $292 \mathrm{~K}$ ensemble. Selecting the quenched lowest-energy structure from the $220 \mathrm{~K}$ trajectory as starting point for a new run turned out to achieve convergence of the overall sampling procedure more quickly than using the $292 \mathrm{~K}$ trajectory. When a convergence (energy of optimized LE structure and stationarity of the PEDF) was achieved, the $292 \mathrm{~K}$ trajectory was used to represent the ensemble of structures under ambient conditions. In total, about $25 \mu \mathrm{s}$ of MD trajectory were propagated.

In order to validate our force field parametrization, we tested it against electronic structure calculations with the PM7 Hamiltonian ${ }^{68}$. The found lowest-energy structures from the $220 \mathrm{~K}$ REMD cycles were reoptimized without constraints using the MOPAC2012 code ${ }^{69}$. The root mean square deviations (RMSD) of all atoms (including hydrogens) of the corresponding optimized structures are reported in table I. These indicate an overall correspondence of the structures with RMSD value between 0.4 and $0.8 \AA$ (the difference between the semiempirical versus force-field optimized structures is visualized for the QSY-A $\beta$-RDM sequence in figure SA1 in the supplemental material) and confirm the expected accuracy of the chromophore-distance prediction of about $1 \AA$.

The semiempirical energetics can also be used to quantify the importance of the different possible tautomers (different charge locations) for the $3+$ and $5+$ systems. The relative PM7 energies for the lowest-energy structures of QSY-A $\beta$-RDM as well as RDM-A $\beta$-QSY in its $3+$ and $5+$ charge states are given in table I. Here the label LYN denotes which of the lysine residues was changed to the neutral form, while HIP denotes the position of the protonated histidine residue in Amber notation. The tautomers differ by at least $0.29 \mathrm{eV}$, so that only the lowest-energy tautomer ensemble was selected for further processing.

\section{Calculation of FRET-efficiencies}

The critical Förster distance for a chromophore pair is calculated as

$$
R_{0}=\left(\frac{9 \ln (10)}{128 \pi^{5} N_{A}} \cdot \frac{\kappa^{2} \Phi_{D}}{n^{4}} \cdot J\right)^{\frac{1}{6}},
$$

where $\Phi_{D}$ is the quantum yield of the donor in the absence of the acceptor, $\mathrm{J}$ the spectral overlap integral, $\mathrm{N}_{A}$
TABLE I. Semiempirical calculations: RMSD values of atomic coordinates for force-field compared to PM7-optimized structures, relative PM7 energies $\Delta \mathrm{E}$ of different PM7optimized tautomers for $3+$ and $5+$ charge states

\begin{tabular}{|c|c|c|}
\hline System & $\operatorname{RMSD}[\AA]$ & $\Delta \mathrm{E}[\mathrm{eV}]$ \\
\hline \multicolumn{3}{|l|}{$\overline{\mathrm{QSY}-\mathrm{A} \beta-\mathrm{RDM}}$} \\
\hline $3+($ LYN 16) & 0.41 & 0.37 \\
\hline $3+($ LYN 28) & 0.65 & 0.00 \\
\hline $4+$ & 0.78 & \\
\hline $5+($ HIP 13$)$ & 0.75 & 0.83 \\
\hline $5+($ HIP 14$)$ & 0.65 & 0.00 \\
\hline $6+$ & 0.59 & \\
\hline \multicolumn{3}{|l|}{ RDM-A $\beta-Q S Y$} \\
\hline $3+($ LYN 16) & 0.53 & 0.00 \\
\hline $3+($ LYN 28) & 0.59 & 0.29 \\
\hline $4+$ & 0.58 & \\
\hline $5+($ HIP 13$)$ & 0.79 & 0.38 \\
\hline $5+($ HIP 14$)$ & 0.48 & 0.00 \\
\hline $6+$ & 0.59 & \\
\hline
\end{tabular}

Avogadro's number, $\mathrm{n}$ the index of refraction being 1 in vacuum, and $\kappa^{2}$ is the orientation factor ${ }^{17,70,71}$. We use a fluorescence quantum yield of 0.82 as known from Rh575 in ethanol ${ }^{72}$.

We first evaluated the sensitivity of FRET-efficiencies in the expected range of chromophore separations as a function of the distance between the optical units of the chromophores and the orientational factor $\kappa^{2}$ utilizing a fixed set of spectral parameters of the two chromophores (parameters are given in table II). The optically active parts of the QSY7 (left part in figure 1) and Rh575 (right part in figure 1) are marked in blue. The geometrical center of these units has been taken to evaluate the chromophore separation. To use the nuclear coordinates to define the center of transition dipole moments is a common strategy (see e.g. Refs. ${ }^{47,48}$ ).

The donor and acceptor spectra were approximated assuming a Gaussian lineshape around absorption and emission maximum $\tilde{\nu}_{\max }$ with the half-width of 1000 $\mathrm{cm}^{-1}$. Equation 3 yields the absorption extinction coefficient $\epsilon_{A}$ in $\mathrm{L} / \mathrm{mol}^{-1} \mathrm{~cm}^{-1}$ from the oscillator strength $\mathrm{f}_{e}^{Q S Y}$ of the acceptor that we have obtained from a TDDFT calculation (see supplemental material), transition energies given in $\mathrm{cm}^{-1}$. The calculated spectra were used to calculate the spectral overlap integral (equation 4 with $F_{D}(\lambda)$ being the normalized fluorescence intensity).

$$
\epsilon_{A}(\tilde{\nu})=\frac{2.175091 \cdot 10^{8}}{\sigma} \cdot f_{e}^{Q S Y} \cdot e^{-2.772\left(\frac{\tilde{\nu}-\tilde{\nu}_{\max }}{\sigma}\right)^{2}}
$$

$$
J(\lambda)=\int_{0}^{\infty} F_{D}(\lambda) \epsilon_{A}(\lambda) \lambda^{4} d \lambda
$$

The numerical results for these chromophoreparameters are visualized in figure 2 (compare also table 
TABLE II. Spectral properties of the QSY7 and RDM chromophores used for FRET-modeling

\begin{tabular}{lll}
\hline variable value & taken from \\
\hline \hline$\lambda_{e m}^{R D M}$ & $505 \mathrm{~nm}$ & gas-phase action spectrum \\
$\lambda_{a b s}^{Q S Y}$ & $539 \mathrm{~nm}$ & gas-phase action spectrum \\
& \\
$\Phi_{D}$ & 0.82 & Ref. $^{72}$ \\
$f_{e}^{Q S Y}$ & 0.85 & this work (TDDFT calculation) $^{\text {Salc }}$ \\
$R_{0}^{\text {calc }}$ & $40.3 \AA$ & this work \\
\hline
\end{tabular}

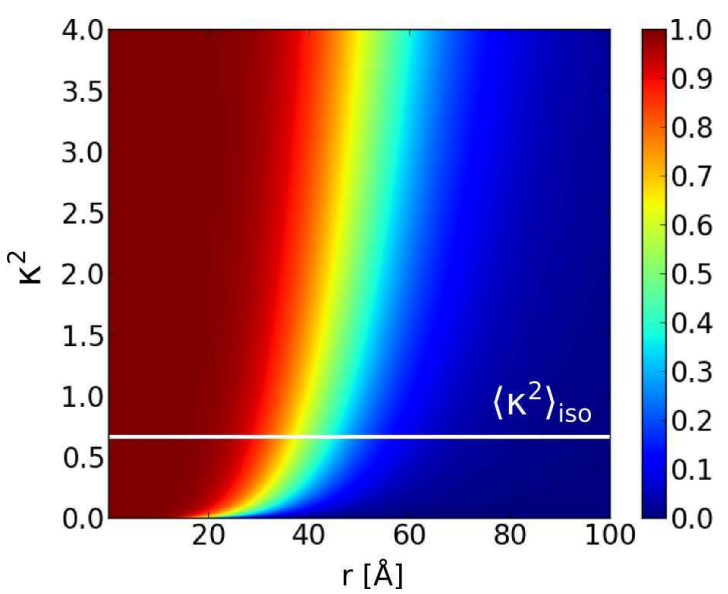

FIG. 2. Calculated FRET efficiency of the QSY7-Rh575 chromophore pair as a function of the chromophore separation and the orientational factor $\kappa^{2}$ (equation 1,2,3,4 with the spectral parameters of QSY7 ad RDM575 in table II). Color coding of $E_{F R E T}$ values according to the legend on the right. The average value of an isotropic orientational distribution $\left\langle\kappa^{2}\right\rangle_{\text {iso }}=2 / 3$ is indicated as a white line.

II). The calculated critical Förster distance $\mathrm{R}_{0}$ for this chromophore pair amounts $40.3 \AA$ (equation 2). The influence of a slightly different fluorescence quantum yield $\left(\Phi_{D}=0.9\right)$ on the final FRET-efficiency values is only marginal $\left(\mathrm{R}_{0}\left[\Phi_{D}=0.9\right]=40.9 \AA\right)$ as is a slightly different oscillator strength $\left(\mathrm{R}_{0}\left[f_{e}=0.95\right]=41.0 \AA\right)$. Within a chromophore separation of $80 \AA$ the FRET efficiency has dropped to nearly zero even when orientation would favor excitation energy transfer (high $\kappa^{2}$ ). On the other hand, the occurrence of high FRET-efficiencies can be substantially decreased by a perpendicular chromophore orientation leading to $\kappa^{2}$ approaching zero. Consequently, if a simple distinction between near and far is to be extended by a more accurate quantitative measure, an inclusion of the orientational effects is mandatory.

In order to predict the average FRET-efficiencies for the chromophore-grafted Amyloid- $\beta$ peptides, we have postprocessed the calculated structural samples in the $3+, 4+, 5+$ and $6+$ charge states and have computed explicitly their FRET-efficiencies. In the following, we compare two different strategies with respect to the structural basis for the FRET-efficiency calculation: a) single low-energy conformations and b) the inclusion of the full canonical ensembles. The results for the modeled FRETefficiencies in comparison with the offset-corrected exper-

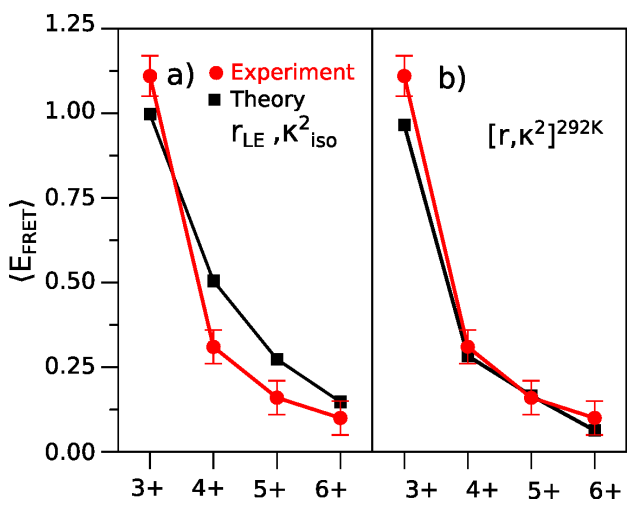

FIG. 3. Explicitly calculated average FRET efficiencies $\left\langle E_{F R E T}\right\rangle$ as a function of charge state: In red the experimental offset-corrected FRET-efficiency is given. $\left\langle E_{F R E T}\right\rangle$ is calculated using a) the chromophore-distance of the lowest energy optimized structure $\mathrm{r}_{L E}$ and $\kappa^{2}=2 / 3$ from an isotropic orientation distribution b) explicit distribution of chromophore-distances as well as orientational factors $\left[\mathrm{r}, \kappa^{2}\right]^{292 K}$. Experimental values are taken from ref. ${ }^{51}$.

imental efficiencies are displayed in figure 3 .

Figure 3a depicts the FRET efficiencies obtained for the lowest-energy structure (LE) with an assumed isotropic chromophore-orientation distribution $\left(\kappa^{2}=\right.$ $2 / 3)$. The agreement of the efficiencies in figure $3 \mathrm{a}$ is qualitative, showing that already the single lowest-energy structure is indicative for the overall trend and may be used to classify chromophores as near or far. Here, thermal effects are not accounted for as the lowest-energy structure corresponds to a temperature of $0 \mathrm{~K}$. This adds to the negligence of chromophore-orientational effects and explains the mediocre agreement.

There are two main strategies to improve the agreement. On the one hand, chromophore-orientations can be explicitly accounted for rather than assuming their isotropic distribution, and, on the other hand, a statistically representative thermal ensemble can be used as a structural basis. We included both effects by using explicit orientational and distance distributions in the 292 $\mathrm{K}$ ensemble and report the resulting $\mathrm{E}_{F R E T}$ values in figure 3b. Evidently, this strategy - which we discuss in detail in the following - reproduces the experimental values in good agreement.

The results from analyzing the thermal ensembles are displayed in figure 4 together with a visualization of a small ensemble of structures. The sampled distance distribution functions as a function of charge state are displayed in figure $4 \mathrm{a}$. The two sequences QSY-A $\beta$-RDM versus RDM-A $\beta$-QSY in the $3+$ charge state exhibit different chromophore separations indicated by the two distinct features of the distribution function. In the $6+$ charge state, three different conformations of the two sequences appear to be sampled at $292 \mathrm{~K}$. In conclusion, a single distance from only one single structure might be a poor representation of the structured and nonsymmetric distance distributions present at $292 \mathrm{~K}$. 

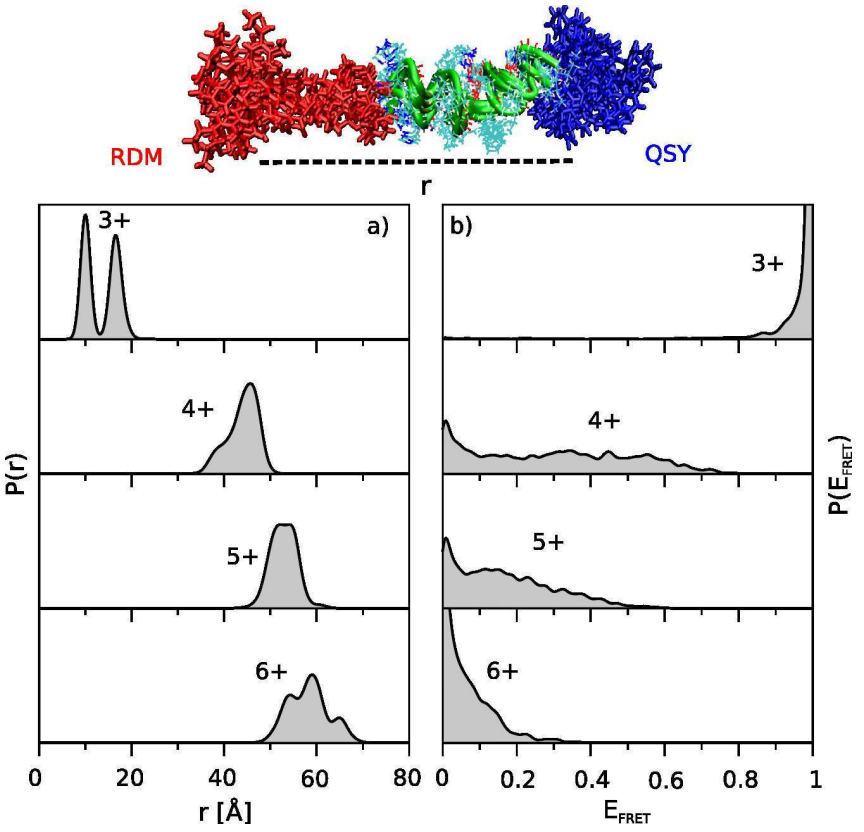

FIG. 4. Normalized probability distribution functions of a) chromophore-distances and b) FRET-efficiencies $\mathrm{E}_{F R E T}$ in the samples of Rh575/QSY7-labeled Amyloid- $\beta$ peptide fragments (smoothed by Gaussians with the half-widths of $1 \AA$ and 0.01 for $\mathrm{r}$ and $\mathrm{E}_{F R E T}$ ) in the charge states $3+, 4+, 5+$ and +6 (from top to bottom). On top, a superposition of 5 structures (blue and red: Rh575 and QSY7 chromophores, green: Amyloid- $\beta$ 12-28) from the $4+$ ensemble visualizes the termal effect.

The orientation factor $\kappa^{2}$ can be calculated from a structure according to equation 5 with the N-atoms of the chromophores defining the transition dipole moment vectors $\mu$ of the optically active units. Upon the sampled canonical ensembles of structures at $292 \mathrm{~K}$ we have calculated the $\kappa^{2}$ distributions shown in figure SA3 in the supplemental material.

$$
\kappa^{2}=\mu_{\mathbf{D}} \cdot \mu_{\mathbf{A}}-3\left(\mu_{\mathbf{D}} \cdot \mathbf{r}\right) \cdot\left(\mu_{\mathbf{A}} \cdot \mathbf{r}\right)
$$

From the distance distributions and the $\kappa^{2}$ distributions we calculated FRET-efficiency distributions in the $292 \mathrm{~K}$ ensembles as shown in figure 4b. These assume a very different functional form compared to the distance distributions. While for the $3+$ and $6+$, the distributions are centrered around a value near to 1 and near to 0 , they are spread over a large interval for the $4+$ and $5+$ charge state. This behavior is originating from the broad shape of the orientational factor $\kappa^{2}$ distribution function with values ranging between 0 and 4 .

From the $\kappa^{2}$ distribution functions, the potential impact of chromophore orientations on the FRETefficiencies can be deduced. For this reason, we quantified the deviation between the observed and the theoretical isotropic $\kappa^{2}$ distribution functions. The integral absolute deviation between the two functions (see supplemental

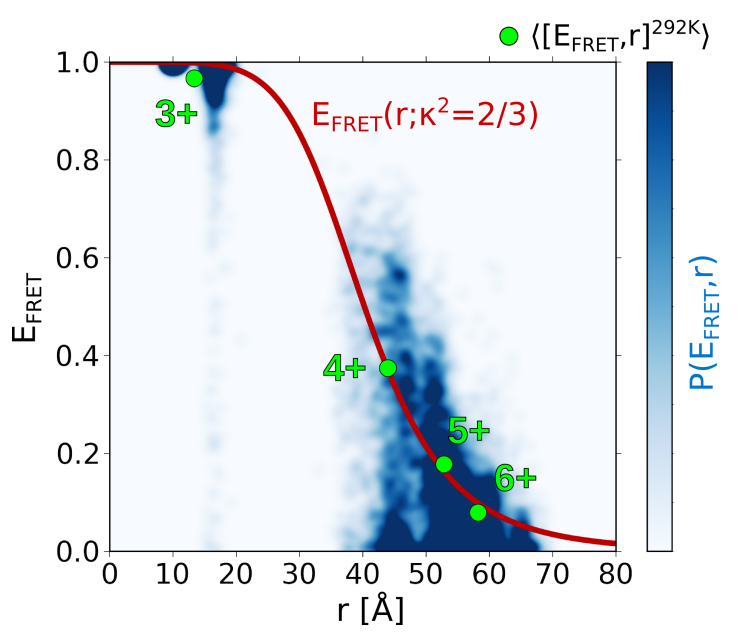

FIG. 5. Correlation between chromophore distance and FRET efficiency for Rh575/QSY7-labeled Amyloid- $\beta$ peptide fragments in the charge range from +3 to +6 . Blue color map shows the normalized probability distribution for the occurrence of a specific $E_{F R E T}, r$ pair in the samples (dark blue regions indicate a high probability). Discrete $E_{F R E T}$ values were calculated for all sampled distance and chromophore orientationsof all charge states and both possible sequences RDM-A $\beta$-QSY and QSY-A $\beta$-RDM and smoothed by twovariate Gaussian functions. The ensemble-average $\mathrm{E}_{F R E T}, \mathrm{r}$ pairs for each charge state are given as green dots. In red, the analytic FRET-efficiency curve is given for a fixed orientation factor of $\kappa^{2}=2 / 3$.

material, figure SA3) amounts to 0.7 for the $3+$ compared to about 0.35 for $4+, 5+$ and $6+$. This high deviation for the $3+$ charge clearly indicates a nonisotropic distribution. In the molecular picture, the narrow $\kappa^{2}$ distribution is associated with discrete conformations being fixed by intramolecular interactions. Notice, that the particular conformation adopted is not only imposed by the overall charge state, but also depends on the charge location (that there exists an interplay between charge distribution, conformation and FRET-efficiencies can be depicted from the different $3+\kappa^{2}$ distributions in figure SA4 of the supplemental material). In the $4+, 5+$ and $6+$ charge systems a quasi-free rotation of the optically active units can be concluded from the $\kappa^{2}$ distributions - where fixing interactions are absent. Overall, the observed decrease in $\mathrm{E}_{F R E T}$ with growing charge state is in agreement with the expectation that the Coulombrepulsion between the charges is the main driving force for the structural changes upon protonation.

For making the overall effect of thermal distributions on FRET-efficiencies more comprehensive, we visualize the $\mathrm{E}_{F R E T}$ versus distance correlation in figure 5. This 2-dimensional plot maps the calculated FRET-efficiencies onto the space of sampled distances. The ensembleaverage values $\left\langle E_{F R E T}\right\rangle$ depicted by the green dots correspond to the values reported in $3 \mathrm{~b}$. This $2 \mathrm{D}$ map demonstrates that there exists no one-to-one mapping of a single distance with a single FRET-efficiency. An 
unambiguous assignment of a single conformation of a molecule to an experimental $\mathrm{E}_{F R E T}$ value is therefore only possible when distance and $\kappa^{2}$ distributions are narrow and posess only one maximum. This limits the isotropic orientational distribution assumption $\left(\kappa^{2}=2 / 3\right)$ often made. Concerning the ensemble-average of chromophore separations at $292 \mathrm{~K}$, however, the $\left(\kappa^{2}=2 / 3\right)$ assumption seems reasonable for the studied system. In conclusion, the knowledge of the explicit thermal structural ensembles is mandatory to perform a structural assignment to a FRET efficiency and to judge assumptions of orientational distributions. For this reason a global experimental-theoretical strategy is the most promising route towards the determination of biomolecular structures in the gas phase.

\section{CONCLUSIONS AND OUTLOOK}

In the presented work, we have performed a complete first-principles modeling of FRET-efficiencies of the chromophore-grafted Amyloid- $\beta$ (12-28) fragment in the charge states $3+, 4+, 5+$ and $6+$. The modeling is based on an extensive exploration of the conformational space of twelve different systems (charge states, tautomers) by Replica-Exchange Molecular Dynamics (REMD) totaling to about $25 \mu \mathrm{s}$ of MD trajectory. We compared the FRET-efficiencies calculated using Förster Theory with measured values from the gas-phase "action"-FRET experiments (Daly et al. ${ }^{51}$ ). By analyzing the agreement between experiment and theory employing different structural representations, we found that an inclusion of distance distributions of the canonical ensemble outperforms calculations where only single low-energy conformations are considered. The former agrees very well with the experimental data. The average FRET-efficiencies of the systems studied here mainly depend on the average chromophore separations and orientational effects do not play an important role. For for the $3+$ charge state however, we have identified fixed chromophore orientations with a reduced rotational freedom which leads to clear non-isotropic $\kappa^{2}$ distribution. The fact that the extent of conformational fixing is depending on the particular system, suggests an explicit inclusion of chromophore orientation distributions into FRET modeling as best practice. Including thermal and conformational effects, we obtain excellent correspondence of theory and experiment, thereby assigning structural parameters such as the interchromophore distance distributions to the experimental signal. An "elongation" of the peptide and the resulting drop in FRET efficiency with increasing charge can therefore be interpreted. The achieved agreement encourages us to use the structural model, parametrization and sampling scheme for further investigations. The simulation protocol can be easily adapted to other peptides charge states and protonation patterns. It has to be noticed, however, that the bottleneck for the numerical accuracy of the procedure is a complete exploration of the molecule's conformational space. In view of the increasing conformational freedom of bigger systems, the presented strategy might be extended with biasing and steering algorithms ${ }^{73}$.

\section{ACKNOWLEDGEMENT}

We gratefully acknowledge computer time granted by the PSMN (Pôle Scientifique de Modélisation Numérique) computing center of ENS de Lyon. A. K acknowledges the German Science Foundation DFG for funding in frame of the Research Fellowship Ku 3251/1-1. The research leading to these results has received funding from the European Research Council under the European Union's Seventh Framework Program (FP7/20072013 Grant agreement N320659).

${ }^{1}$ C. M. Dobson, Nature 426, 884 (2003).

${ }^{2}$ B. T. Ruotolo and C. V. Robinson, Curr. Opin. Chem. Biol. 10, 402 (2006).

${ }^{3}$ R. H. van den Heuvel and A. J. R. Heck, Curr. Opin. Chem. Biol. 8, 519 (2004)

${ }^{4}$ T. Förster, Annalen der Physik 2, 55 (1948).

${ }^{5}$ G. D. Scholes, Annu. Rev. Phys. Chem. 54, 57 (2003).

${ }^{6}$ L. Stryer and R. P. Gaughland, Proc. Natl. Acad. Sci. U. S. A. 58, 719 (1967).

${ }^{7}$ S. Kalanin, S. Felekyan, A. Valeri, and C. A. M. Seidel, J. Phys. Chem. B. 112, 8361 (2008).

${ }^{8}$ H. Neubauer, N. Gaiko, S. Berger, J. Schaffer, C. Eggeling, J. Tuma, L. Verdier, C. A. M. Seidel, C. Griesinger, and A. Volkmer, J. Am. Chem. Soc. 129, 12746 (2007).

${ }^{9}$ A. K. Woźniak, S. Nottrott, E. Kühn-Hölsken, G. F. Schröder, H. Grubmüller, R. Lührmann, C. A. Seidel, and F. Oesterhelt, RNA 11, 1545 (2005).

${ }^{10}$ S. Sindbert, S. Kalinin, H. Nguyen, A. Kienzler, L. Clima, W. Bannwarth, B. Appel, S. Müller, and C. A. M. Seidel, J. Am. Chem. Soc. 133, 2463 (2011).

${ }^{11}$ B. Wieb van der Meer, G. Coker III, and S. Y. S. Chen, Resonance Energy Transfer: Theory and Data, 1 edition (John Wiley \& Sons, 1994).

${ }^{12}$ A. K. Woźniak, G. F. Schröder, H. Grubmüller, C. A. M. Seidel, and F. Oesterhelt, Proc. Natl. Acad. Sci. U. S. A. 105, 18337 (2008).

13 A. T. Brunger, P. Strop, M. Vrljic, S. Chu, and K. R. Weninger, J. Struct. Biol. 173, 497 (2011).

${ }^{14}$ M. G. Düser, Y. Bi, N. Zarrabi, S. D. Dunn, and M. Börsch, J. Biol. Chem. 283, 33602 (2008).

${ }^{15}$ G. F. Schröder and H. Grubmüller, Comput. Phys. Commun. 158, 150 (2004).

${ }^{16}$ S. Uphoff, S. J. Holden, L. L. Reste, J. Periz, S. van de Linde, M. Heilemann, and A. N. Kapanidis, Nat. Methods 7, 831 (2010).

${ }^{17}$ E. Sisamakis, A. Valeri, S. Kalinin, P. J. Rothwell, and C. A. Seidel, in Single Molecule Tools, Part B: Super-Resolution, Particle Tracking, Multiparameter, and Force Based Methods, Methods in Enzymology, Vol. 475, edited by N. G. Walter (Academic Press, 2010) pp. $455-514$.

${ }^{18}$ T. Ha, T. Enderle, D. F. Ogletree, D. S. Chemla, P. R. Selvin, and S. Weiss, Proc. Natl. Acad. Sci. U. S. A. 93, 6264 (1996).

${ }^{19}$ E. B. Shera, N. K. Seitzinger, L. M. Davis, R. A. Keller, and S. A. Soper, Chem. Phys. Lett. 174, 553 (1990).

${ }^{20}$ A. J. Berglund, A. C. Doherty, and H. Mabuchi, Phys. Rev. Lett. 89, 068101 (2002).

${ }^{21}$ S. Weiss, Science 283, 1676 (1999).

${ }^{22}$ P. R. Selvin, Nat. Struct. Biol. 7, 730 (2000). 
${ }^{23}$ B. Schuler, E. A. Lipman, and W. A. Eaton, Nature 419, 743 (2002).

${ }^{24}$ B. N. G. Giepmans, A. S. R., M. H. Ellisman, and R. Y. Tsien, Science 312, 217 (2006).

${ }^{25}$ A. S. Danell and J. H. Parks, Int. J. Mass Spectrom. 229, 35 (2003).

${ }^{26}$ M. Dashtiev, V. Azov, V. Frankevich, L. Scharfenberg, and R. Zenobi, J. Am. Soc. Mass Spectrom. 16, 1481 (2005).

${ }^{27}$ F. O. Talbot, A. Rullo, H. Yao, and R. A. Jockusch, J. Am. Chem. Soc. 132, 16156 (2010).

${ }^{28}$ V. Frankevich, X. W. Guan, M. Dashtiev, and R. Zenobi, Eur. J. Mass Spectrom. 11, 475 (2005).

${ }^{29}$ A. T. Iavarone, J. Meinen, S. Schulze, and J. H. Parks, Int. J. Mass Spectrom. 253, 172 (2006).

${ }^{30}$ A. T. Iavarone, A. Patriksson, D. van der Spoel, and J. H. Parks, J. Am. Chem. Soc. 129, 6726 (2007).

${ }^{31}$ X. G. Shi, D. Duft, and J. H. Parks, J. Phys. Chem. B 112, 12801 (2008)

${ }^{32}$ V. Frankevich, V. Chagovets, F. Widjaja, K. Barylyuk, Z. Y. Yang, and R. Zenobi, Phys. Chem. Chem. Phys. 16, 8911 (2014).

${ }^{33}$ N. G. Hendricks, N. M. Lareau, . M. Stow, J. A. McLean, and R. R. Julian, J. Am. Chem. Soc. 136, 13363 (2014).

${ }^{34}$ V. Frankevich, V. Chagovets, F. Widjaja, K. Barylyuk, Z. Yang, and R. Zenobi, Phys. Chem. Chem. Phys. 16, 8911 (2014).

${ }^{35}$ S. Daly, F. Poussigue, A.-L. Simon, L. MacAleese, F. Bertorelle, F. Chirot, R. Antoine, and P. Dugourd, Anal. Chem. 86, 8798 (2014).

${ }^{36}$ A. Miranker, C. V. Robinson, S. E. Radford, R. T. Aplin, and C. M. Dobson, Science 262, 896 (1993).

${ }^{37}$ A. J. R. Heck, Nat. Methods 5, 927 (2008).

${ }^{38}$ G. Vonhelden, T. Wyttenbach, and M. T. Bowers, Science 267, 1483 (1995)

${ }^{39}$ A. Iqbal, S. Arslan, B. Okumus, T. J. Wilson, G. Giraud, D. G. Norman, and T. Ha, Science 105, 2 (2008).

${ }^{40}$ F. D. Lewis, L. Zhang, and X. Zuo, J. Am. Chem. Soc. 127, $10002(2005)$.

${ }^{41}$ D. B. VanBeek, M. C. Zwier, J. M. Shorb, and B. P. Krueger, Biophys. J. 92, 4168 (2007).

42Z.-Q. You and C.-P. Hsu, Int. J. Quant. Chem. 104, 102 (2014).

${ }^{43}$ R. Mitrić, J. Petersen, and V. Bonačić-Koutecký, "Multistate nonadiabatic dynamics on the fly in complex systems and its control by laser fieldes," in Conical Intersections (2011) Chap. 13, pp. 497-568.

${ }^{44}$ Y. Okuno and S. Mashiko, Int. J. Quant. Chem. 94, 36 (2003).

${ }^{45} \mathrm{~J}$. Megow, B. Röder, A. Kulesza, V. Bonačić-Koutecký, and V. May, ChemPhysChem 12, 645 (2011).

${ }^{46} \mathrm{~J}$. Megow, T. Renger, and V. May, ChemPhysChem 15, 478 (2014).

${ }^{47}$ M. Hoefling, N. Lima, D. Haenni, C. A. M. Seidel, B. Schuler, and H. Grubmüller, PLoS ONE 6, e19791 (2011).

${ }^{48}$ M. Hoefling and H. Grubmüller, Comp. Phys. Commun. 184, 841 (2013)

${ }^{49}$ Y. Sugita and Y. Okamoto, Chem. Phys. Lett. 314, 141 (1999).

${ }^{50}$ F. Chirot, F. Calvo, , F. Albrieux, J. Lemoine, Y. O. Tsybin, and P. Dugourd, J. Am. Soc. Mass Spectrom. 23, 386 (2012).

${ }^{51}$ S. Daly, A. Kulesza, F. Poussigue, A.-L. Simon, C. M. Choi, G. Knight, F. Chirot, L. MacAleese, R. Antoine, and P. Dugourd, Chem. Sci., DOI:10.1039/C5SC01436H (2015).

${ }^{52}$ T. N. Le, J. C. Poully, F. Lecomte, N. Nieuwjaer, B. Manil, C. Desfrançois, F. Chirot, J. Lemoine, P. Dugourd, G. van der Rest, and G. Grégoire, J. Am. Soc. Mass Spectrom. 24, 1937 (2013).
${ }^{53}$ N. S. de Groot, F. X. Aviles, J. Vendrell, and S. Ventura, FEBS J. 273, 658 (2006).

${ }^{54}$ A. Baumketner, M. G. Krone, and J.-E. Shea, Proc. Natl. Acad. Sci. U. S. A. 105, 6027 (2008).

${ }^{55}$ K. Murakami, K. Irie, A. Morimoto, H. Ohigashi, M. Shindo, M. Nagao, T. Shimizu, and T. Shirasawa, J. Biol. Chem. 278, 46179 (2003)

${ }^{56}$ Q. Enjalbert, M. Girod, R. Simon, J. Jeudy, F. Chirot, A. Salvador, R. Antoine, P. Dugourd, and J. Lemoine, J. Anal. Bioanal. Chem. 405, 2321 (2013).

${ }^{57}$ M. W. Forbes and R. A. Jockusch, J. Am. Soc. Mass Spectrom. 22, 93 (2011).

${ }^{58}$ J. Wang, P. Cieplak, and P. A. Kollman, J. Comp. Chem. 21, 1049 (2000).

${ }^{59}$ J. Wang, R. M. Wolf, J. W. Caldwell, P. A. Kollman, and D. A. Case, J. Comp. Chem. 25, 1157 (2004).

${ }^{60}$ M. J. S. Dewar, E. G. Zoebisch, E. F. Healy, and J. J. P. Stewart, J. Am. Chem. Soc. 107, 3902 (1985).

${ }^{61}$ A. Jakalian, B. L. Bush, D. B. Jack, and C. I. Bayly, J. Comp. Chem. 21, 132 (2000).

${ }^{62}$ A. Sousa da Silva and W. Vranken, BMC Research Notes 5, 367 (2012).

63 J. Wang, W. Wang, P. A. Kollman, and D. A. Case, J. Mol. Graph. Model. 25, 247 (2006).

${ }^{64}$ B. Hess, C. Kutzner, D. van der Spoel, and E. Lindahl, J. Chem. Theory Comput. 4, 435 (2008).

${ }^{65}$ D. Van Der Spoel, E. Lindahl, B. Hess, G. Groenhof, A. E. Mark, and H. J. C. Berendsen, J. Comput. Chem. 26, 1701 (2005).

${ }^{66}$ H. Berendsen, Comp. Phys. Commun. 91, 43 (1995)

${ }^{67}$ G. Bussi, D. Donadio, and M. Parrinello, J. Chem. Phys. 126, $014101+(2007)$.

${ }^{68}$ J. J. P. Stewart, J. Mol. Model. 19, 1 (2013).

${ }^{69}$ J. J. P. Stewart, "Mopac2012, stewart computational chemistry, colorado springs, co, usa," http://OpenMOPAC.net (2012).

${ }^{70}$ B. Schuler, in Protein Folding Protocols, Methods in Molecular Biology, Vol. 350, edited by Y. Bai and R. Nussinov (Humana Press, 2006) pp. 115-138.

${ }^{71}$ S. E. Braslavsky, E. Fron, H. B. Rodriguez, E. S. Roman, G. D. Scholes, G. Schweitzer, B. Valeur, and J. Wirz, Photochem. Photobiol. Sci. 7, 1444 (2008).

${ }^{72}$ F. L. Arbeloa, T. L. Arbeloa, E. G. Lage, I. L. Arbeloa, and F. C. Deschryver, J. Photochem. Photobiol., A 56, 313 (1991).

${ }^{73}$ F. Calvo, F. Chirot, F. Albrieux, J. Lemoine, Y. O. Tsybin, P. Pernot, and P. Dugourd, J. Am. Soc. Mass Spectrom. 23, 1279 (2012)

See supplemental material at [URL will be inserted by AIP] for an overlay of structures optimized with our force-field parametrization and the semiempirical PM7 level of theory; a detailed analysis of the influence of thermal effects for the distance and orientational distributions on the predicted FRET-efficiency; numerical values of the calculated FRET-efficiencies; inter-chromophore distance and orientational factor distributions of chromophore-grafted Amyloid- $\beta$ 12-28; orientational factor distributions for a different tautomer (charge location) of the $3+$ chromophore-grafted Amyloid- $\beta$ 12-28; details of DFT/TDDFT calculations to obtain the QSY7 absorption oscillator strength and nonstandard GAFF/AMBER99 residue libraries in Amber format for Ac-QSY, Ac-RDM, QSY-NH $\mathrm{N}_{2}$ and RDM-NH residues. 
Supplemental Material for:

Structural exploration and Förster theory modeling for the interpretation of gas-phase FRET measurements: Chromophore-grafted Amyloid- $\beta$ peptides

Alexander Kulesza, Steven Daly, Luke MacAleese, Rodolphe Antoine, Philippe Dugourd* Institut Lumière Matière, UMR5306 Université Claude Bernard Lyon 1-CNRS , Université de Lyon, 69622 Villeurbanne cedex, France

E-mail: philippe.dugourd@univ-lyon1.fr

*To whom correspondence should be addressed 


\section{Assessment of parametrization}

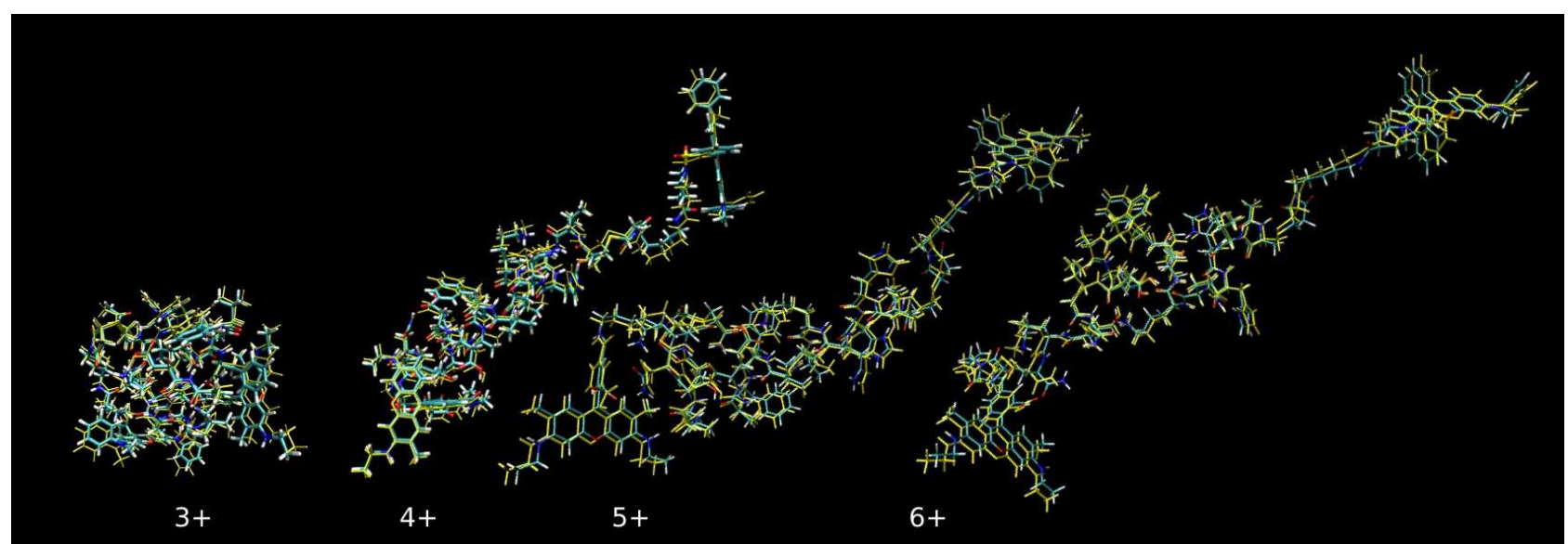

Figure SA1: Overlay of optimized structures of QSY-A $\beta-R D M$. The force-field optimized structure is given in yellow, while the PM7 optimized structure is given color-coded for the atom type.

\section{Influence of thermal and orientational effects for FRET modeling}

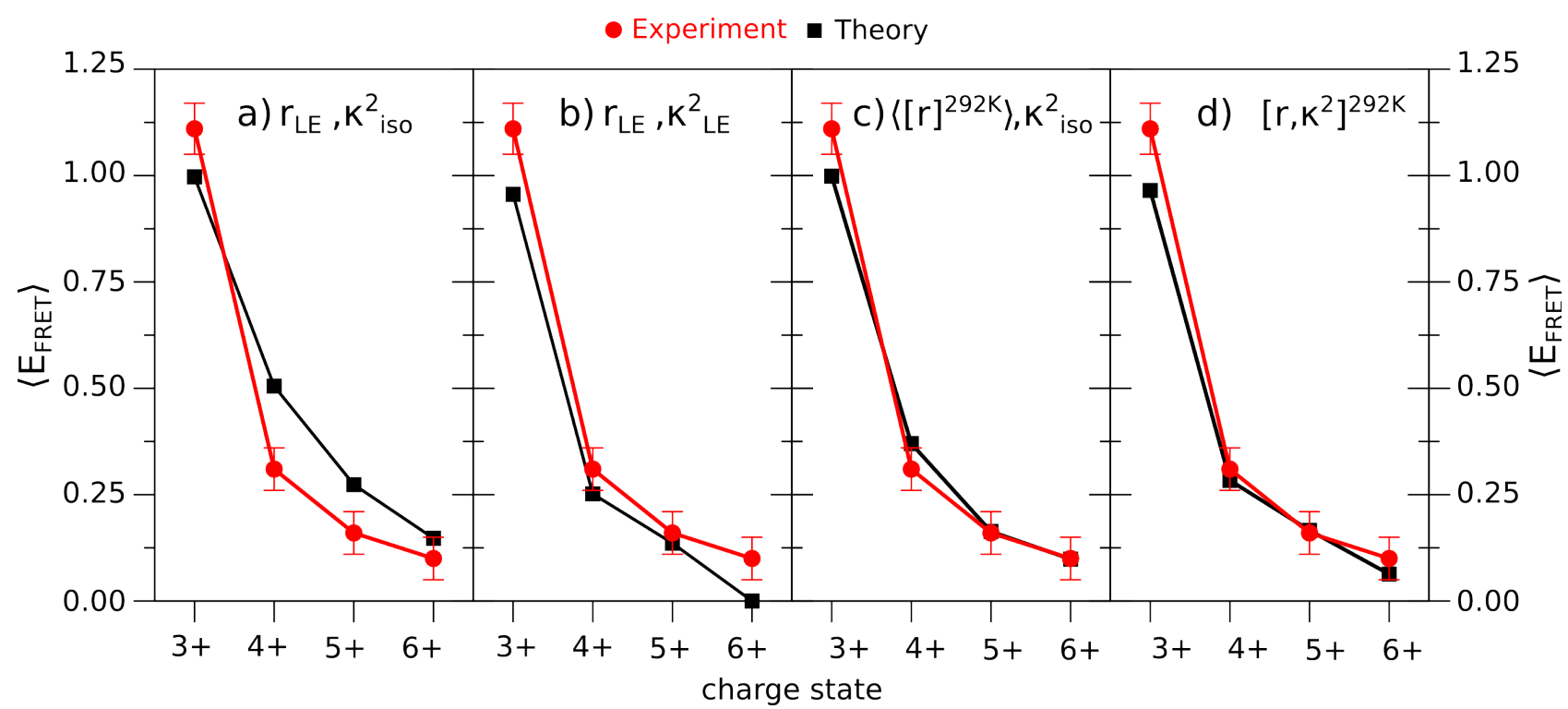

Figure SA2: Explicitly calculated average FRET efficiencies $\left\langle E_{F R E T}\right\rangle$ as a function of charge state: In red the experimental offset-corrected FRET-efficiency is given. $\left(E_{F R E T}\right\rangle$ is calculated using a) the chromophore-distance of the lowest energy optimized structure $r_{L E}$ and $\kappa^{2}=2 / 3 b$ ) the chromophoredistance of the lowest energy structure $r_{L E}$ and the actual value of $\kappa^{2}$ for the lowest-energy structure, c) the average from the thermal distribution of chromophore-distances $\left\langle[r]^{292 K}\right\rangle$ and $\left.\kappa^{2}=2 / 3 d\right)$ explicit distribution of chromophore-distances as well as orientational factors $\left[r, K^{2}\right]^{292 K}$. For values see table SA1.Experimental values are taken from S. Daly et al., to be published (2015). 
Table SA1: Calculated average FRET efficiencies $\left\langle E_{F R E T}\right\rangle$ as a function of charge state using a) the chromophore-distance of the lowest energy optimized structure $r_{L E}$ and $\kappa^{2}=2 / 3, b$ ) the chromophoredistance of the lowest energy structure $r_{L E}$ and the actual value of $\kappa^{2}$ for the lowest-energy structure, c) the average from the thermal distribution of chromophore-distances $\left\langle[r]^{292 K}\right\rangle$ and $\left.\kappa^{2}=2 / 3 d\right)$ explicit distribution of chromophore-distances as well as orientational factors $\left[r, K^{2}\right]^{292 K}$.

\begin{tabular}{|l|l|l|l|l|}
\hline \multicolumn{1}{c}{} & a) & b) & c) & d) \\
\hline $3+$ & 1.00 & 0.96 & 1.00 & 0.96 \\
\hline $4+$ & 0.51 & 0.25 & 0.37 & 0.29 \\
\hline $5+$ & 0.27 & 0.14 & 0.16 & 0.17 \\
\hline $6+$ & 0.15 & 0.00 & 0.10 & 0.06 \\
\hline
\end{tabular}

Figure SA2 shows the influence of distance distributions and orientational distributions on the FRET-efficiency predictions. SA2a) and SA2b) correspond to figure 3a and $3 b$ in the main text (taking the lowest-energy optimized structure and isotropic orientational distribution / explicit thermal distance and orientational distributions) Figure SA2b) shows the FRET-efficiency values from the lowest-energy optimized structures but with the explicit $\kappa^{2}$ value for the structure. Although the results for the $4+$ and $5+$ appear to better fit the experiment than the calculation in figure 3a/SA2a, a significant error cancellation might be present (QSY-A $\beta$-RDM and RDM-A $\beta$ QSY FRET efficiencies differ by up to 0.2 ) The deviation of the $6+$ charge state from the experiment is characteristic for a situation in which such cancellation does not take place. Therefore, we regard calculating FRET efficiencies based on a single e.g. LE structure but accounting its explicit chromophore-orientation inferior to using $\mathrm{k}^{2}=2 / 3$. In SA2c) a thermal ensemble of distances corresponding to $292 \mathrm{~K}$ is used for the calculation. Here, the width and shape of the distance distribution function is accounted for in its influence on the FRET-efficiencies. Chromophore-orientations have been assumed to be isotropically distributed. This treatment proves to be rather accurate for the systems studied here, as a very good quantitative agreement with the experiment is achieved. Comparing figure SA2c with SA3d (figure 3b in the main text) indicates only a slight improvement of the numerical results when also explicit orientational distributions are included. Although the $3+$ charge state displays fixed conformations with narrow $\mathrm{K}^{2}$ distribution, it does not greatly affect the averaged FRET-efficiencies. The low chromophore distance is responsible for FRET-efficiencies that are very near to one, irrespective of their orientation. It has to be pointed out that for a different system, conformational fixing could coincide with separations in the sensitive region (e.g . if conformational fixing would be existent in the $5+$ charge state of our system) which would lead to a breakdown of the good agreement with the strategy of figure SA3c. 


\section{Analysis of distance distribution and chromophore orientation distribution}

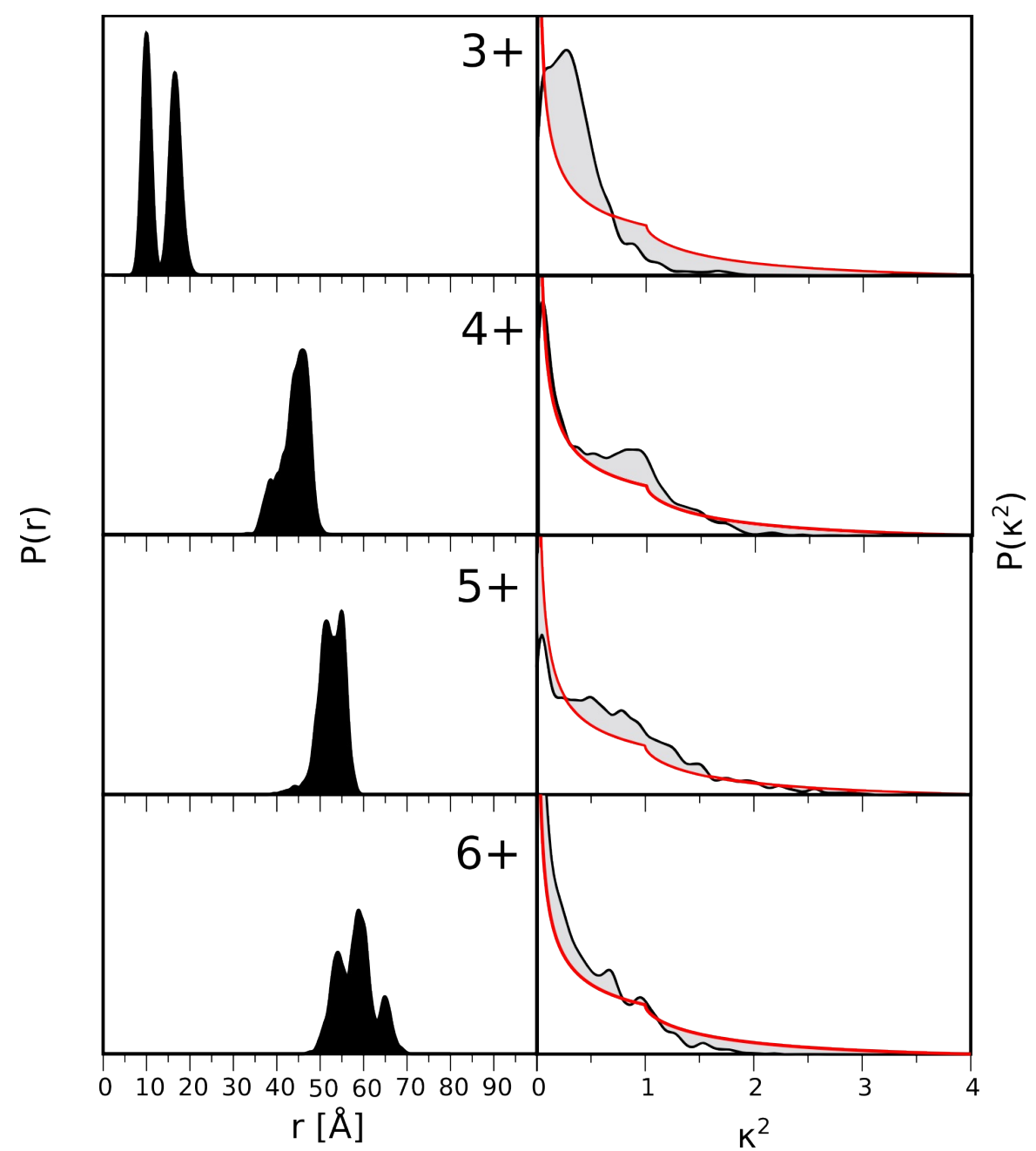

Figure SA3: Normalized probability distribution functions for chromophore-chromophore distance $r$ (left) and orientational factor $\kappa^{2}$ (right) in the structure samples (292 K) of chromophore-grafted Amyloid- $\beta$ fragments in the charge states $3+$ to $6+$ (smoothing by Gaussian functions with halfwidths of $1 \AA$ and 0.05). A 50/50 mixture of the two sequences QSY-A $\beta-R D M$ and RDM-A $\beta-Q S Y$ is assumed. The red curve in the $\kappa^{2}$ depicts ideal isotropic distribution of the transition dipole vectors. Differences in the sampled $\kappa^{2}$ distribution functions from isotropic limit are marked in gray. 


\section{Analysis of orientational factors as a function of charge distribution}

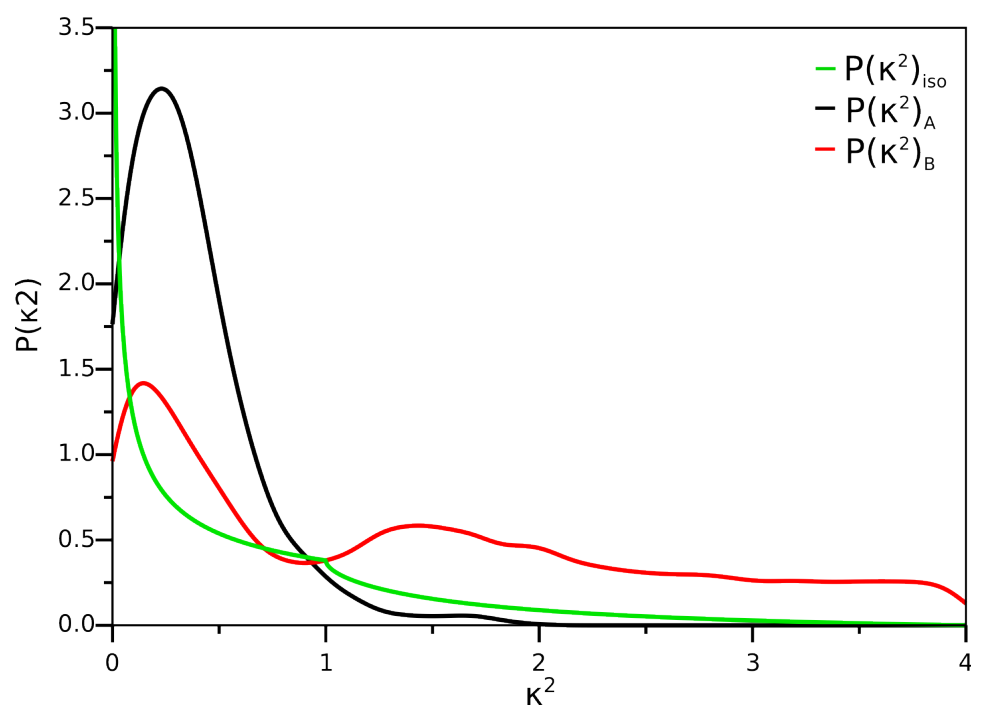

Figure SA4: Normalized probability distribution functions for orientational factors $\kappa^{2}$ in the converged thermal ensembles for chromophore-grafted Amyloid- $\beta$ in the charge state $3+$ compared to isotropic distribution (green). The black curve $P\left(\kappa^{2}\right)_{A}$ is shown for the lowest-energy proton location where QSY-A $\beta-R D M(L Y N$ 28) and RDM-A $\beta-Q S Y$ (LYN 16) are present (see main text and table 2) while $P\left(\kappa^{2}\right)_{B}$ denotes the configurations $Q S Y-A \beta-R D M(L Y N 16)$ and $R D M-A \beta-Q S Y$ (LYN 28), which are higher in energy by 0.37 and $0.29 \mathrm{eV}$, respectively, and therefore not expected to be significantly populated at $293 \mathrm{~K}$. Notice that $P\left(K^{2}\right)_{A}$ is identical to the one shown in figure SA3 (upper part).

\section{Calculation of QSY7 absorption oscillator strength}

The absorption oscillator strength was estimated from a TDDFT calculation of the S1 state of a QSY7 dye. We obtained a low-energy conformation of the molecule by running simulated annealing coupled to molecular dynamics where we used the semiempirical AM1 Hamiltonian [1] for the forces. This structure was optimized at the B3LYP/6-31G** level of density functional theory [2-5] and applying Grimme's D3BJ dispersion correction [6]. The S1 $\leftarrow$ S0 transition properties were calculated with the CAM-B3LYP functional [7] and using the $6-311++\mathrm{G}^{* *}$ basis set. All calculations were performed using Gaussian09 [8].

\section{Nonstandard residue parameter files in Amber format}

AC-QSY-Nterm.lib - library for the N-terminal QSY residue AC-RDM-Nterm.lib - library for the N-terminal RDM residue SupplementalMaterial.CHROMOPHORES-AMBERLIB.frcmod - additional parameters from the Antechamber procedure and used Amber-GAFF interface QSY-NH2-Cterm.lib - library for the C-terminal QSY residue RDM-NH2-Cterm.lib - library for the C-terminal RDM residue 


\section{References}

[1] M. J. S. Dewar, E. G. Zoebisch, E. F. Healy, J. J. P. Stewart, J. Am. Chem. Soc. 107 (1985), 3902-3909.

[2] A.D. Becke, J.Chem.Phys. 98 (1993) 5648-5652

[3] C. Lee, W. Yang, R.G. Parr, Phys. Rev. B 37 (1988) 785-789

[4] S.H. Vosko, L. Wilk, M. Nusair, Can. J. Phys. 58 (1980) 1200-1211

[5] P.J. Stephens, F.J. Devlin, C.F. Chabalowski, M.J. Frisch, J.Phys.Chem. 98 (1994) 11623-11627

[6] S. Grimme, S. Ehrlich and L. Goerigk, J. Comp. Chem. 32 (2011) 1456-65.

[7] T. Yanai, D. Tew, and N. Handy, Chem. Phys. Lett., 393 (2004) 51-57.

[8] Gaussian 09, Revision D.01, M. J. Frisch, G. W. Trucks, H. B. Schlegel, G. E. Scuseria, M. A. Robb, J. R. Cheeseman, G. Scalmani, V. Barone, B. Mennucci, G. A. Petersson, H. Nakatsuji, M. Caricato, X. Li, H. P. Hratchian, A. F. Izmaylov, J. Bloino, G. Zheng, J. L. Sonnenberg, M. Hada, M. Ehara, K. Toyota, R. Fukuda, J. Hasegawa, M. Ishida, T. Nakajima, Y. Honda, O. Kitao, H. Nakai, T. Vreven, J. A. Montgomery, Jr., J. E. Peralta, F. Ogliaro, M. Bearpark, J. J. Heyd, E. Brothers, K. N. Kudin, V. N. Staroverov, R. Kobayashi, J. Normand, K. Raghavachari, A. Rendell, J. C. Burant, S. S. Iyengar, J. Tomasi, M. Cossi, N. Rega, J. M. Millam, M. Klene, J. E. Knox, J. B. Cross, V. Bakken, C. Adamo, J. Jaramillo, R. Gomperts, R. E. Stratmann, O. Yazyev, A. J. Austin, R. Cammi, C. Pomelli, J. W. Ochterski, R. L. Martin, K. Morokuma, V. G. Zakrzewski, G. A. Voth, P. Salvador, J. J. Dannenberg, S. Dapprich, A. D. Daniels, O. Farkas, J. B. Foresman, J. V. Ortiz, J. Cioslowski, and D. J. Fox, Gaussian, Inc., Wallingford CT, 2009. 